\title{
Phronesis
}

\section{Appréhender le développement d'un discours d'expérience sur le travail en accompagnement VAE : vers une analyse des mouvements dialogiques, relationnels, contextuels et interprétatifs \\ Developing discourses of experience about work within the practices of guidance for the Accreditation of Prior Learning : An analysis of dialogical, relational, contextual and interpretive movements}

\section{Vanessa Rémery}

Volume 5, numéro 3-4, 2016

Quels discours pour quel développement professionnel ?

URI : https://id.erudit.org/iderudit/1039089ar

DOI : https://doi.org/10.7202/1039089ar

Aller au sommaire du numéro

\section{Éditeur(s)}

Université de Sherbrooke

Champ social éditions

ISSN

1925-4873 (numérique)

Découvrir la revue

Citer cet article

Rémery, V. (2016). Appréhender le développement d'un discours d'expérience sur le travail en accompagnement VAE : vers une analyse des mouvements dialogiques, relationnels, contextuels et interprétatifs. Phronesis, 5(3-4), 100-112. https://doi.org/10.7202/1039089ar

\section{Résumé de l'article}

La contribution rend compte des résultats d'une recherche conduite dans un organisme de formation aux métiers socio-éducatifs auprès de plusieurs conseillers en Validation des Acquis de l'Expérience. A partir d'une observation ethnographique, nous nous intéressons à l'activité de ces conseillers au cours des entretiens menés avec les candidats qu'ils accompagnent. Plus spécifiquement, nous cherchons à comprendre comment l'activité qu'ils déploient peut soutenir le développement par le candidat d'un discours d'expérience sur son travail. La recherche s'intéresse à la fois aux transformations des énoncés d'expérience produits par le candidat, mais aussi aux modalités par lesquelles ce qui se dit à propos du travail se transforme dans le dialogue qui s'engage entre le candidat et le conseiller. La question de la contribution du conseiller est centrale pour comprendre le développement du discours d'expérience dans ce contexte. Nous proposons ici des éléments de réponse à cette problématique à partir de l'analyse d'extraits d'entretien en mettant en évidence la façon dont peuvent être repérés différents types de mouvements dans les échanges conseiller/candidat contribuant aux dynamiques développementales en jeu de l'entretien. 


\title{
Appréhender le développement d'un discours d'expérience sur le travail en accompagnement VAE : vers une analyse des mouvements dialogiques, relation- nels, contextuels et interprétatifs
}

\author{
Vanessa RÉMERY
}

\author{
Laboratoire RIFT, Équipe Interaction \& Formation \\ Université de Genève \\ 42 bd du Pont d'Arve \\ 1211 Genève 4, Suisse \\ Vanessa.Remery@unige.ch
}

Mots clés : Validation des Acquis de l'Expérience; Accompagnement; Discours d'expérience sur le travail ; Développement.

Résumé : La contribution rend compte des résultats d'une recherche conduite dans un organisme de formation aux métiers socioéducatifs auprès de plusieurs conseillers en Validation des Acquis de l'Expérience. A partir d'une observation ethnographique, nous nous intéressons à l'activité de ces conseillers au cours des entretiens menés avec les candidats qu'ils accompagnent. Plus spécifiquement, nous cherchons à comprendre comment l'activité qu'ils déploient peut soutenir le développement par le candidat d'un discours d'expérience sur son travail. La recherche s'intéresse à la fois aux transformations des énoncés d'expérience produits par le candidat, mais aussi aux modalités par lesquelles ce qui se dit à propos du travail se transforme dans le dialogue qui s'engage entre le candidat et le conseiller. La question de la contribution du conseiller est centrale pour comprendre le développement du discours d'expérience dans ce contexte. Nous proposons ici des éléments de réponse à cette problématique à partir de l'analyse d'extraits d'entretien en mettant en évidence la façon dont peuvent être repérés différents types de mouvements dans les échanges conseiller/candidat contribuant aux dynamiques développementales en jeu de l'entretien.

Title : Developing discourses of experience about work within the practices of guidance for the Accreditation of Prior Learning : An analysis of dialogical, relational, contextual and interpretive movements

Keyword : Accreditation of Prior Learning ; Guidance ; Experience discourse about work ; Development.

\begin{abstract}
The contribution describes the results of a research on the work activity of counselors in the field of Accreditation of Prior Learning. From an ethnographic observations conducted in a training Social Work organization, we analyse the activity of the counselors during the interviews with the candidates they guide. We seek to understand how the activity they deploy can support the candidate's development of an experience discourse about his or her work. The research focuses both on the transformations of the candidate's discourses of experience and on the ways in which what is said about work is transformed within the dialogue between the candidate and the counselor. The question of the counselor's contribution is central to understanding the development of the discourse of experience in this context. We propose here some answers to this problem based on the analysis of real dialogues by highlighting how different types of movements can be identified in the counselor / candidate exchanges contributing to the developmental dynamic observed.
\end{abstract}




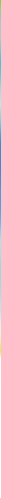

\section{Introduction}

Le dispositif de Validation des Acquis de l'Expérience (VAE), tel qu'il est mis en œuvre en France, permet à toute personne qui se reconnait une expérience professionnelle et/ou sociale de la faire valider socialement dans un cadre législatif défini. Pour user de son droit à la reconnaissance des acquis de son expérience, le candidat doit faire la preuve de ceux-ci. Contrairement aux dispositifs traditionnels de certification qui privilégient davantage une évaluation des compétences des formés à restituer les savoirs légitimés et enseignés par les institutions de formation, la VAE sanctionne la production d'un discours d'expérience sur le travail (Barbier, 2006). Le candidat doit être en mesure de rendre compte de son activité de travail, de l'analyser à la lumière de son expérience, de préparer un dossier et de le faire valoir à l'occasion d'un entretien avec un jury. Comment ce discours s'élabore-t-il dans le cadre des entretiens d'accompagnement dont le candidat peut bénéficier pour le soutenir dans sa démarche VAE?

La contribution vise à rendre compte des résultats d'une recherche conduite dans un organisme de formation aux métiers socio-éducatifs auprès de plusieurs conseillers VAE. A partir d'une observation ethnographique, nous nous sommes intéressée à l'activité de ces conseillers au cours des entretiens menés avec les candidats qu'ils accompagnent. Plus spécifiquement, nous avons cherché à comprendre comment l'activité qu'ils déploient peut soutenir le développement par le candidat d'un discours d'expérience sur son travail. La recherche s'est intéressée à la fois aux transformations des énoncés d'expérience produits par le candidat, mais aussi aux modalités par lesquelles ce qui se dit à propos du travail se transforme dans le dialogue qui s'engage entre le candidat et le conseiller. En effet, loin d'adopter une position de retrait des interprétations proposées par le candidat, le conseiller se positionne, évalue, complète, reformule, etc. La question de la contribution du conseiller est centrale pour comprendre le développement du discours d'expérience dans ce contexte. Nous proposons ici des éléments de réponse à cette problématique à partir de l'analyse d'extraits d'entretien en mettant en évidence la façon dont peuvent être repérés différents types de mouvements dans les échanges conseiller/candidat contribuant aux dynamiques développementales en jeu de l'entretien.

\section{Instrumentation du dialogue et développement d'un discours d'expérience sur le travail}

La situation d'accompagnement place le langage au cœur de l'activité du conseiller et du candidat. Comme toute forme d'entretien, l'entretien en situation d'accompagnement VAE est avant tout un dialogue et c'est de ses propriétés de dialogue qu'il tire sa spécificité (Salazar-Orvig \& Grossen, 2008). C'est une ressource à la fois pour le conseiller et le candidat dans l'accomplissement de leur activité. On peut même dire que ce type d'entretien relève d'une activité principalement médiatisée par le langage qui mobilise des compétences à dire, à expliciter, à rendre compte, à décrire, à analyser, à argumenter, etc. Du point de vue du candidat, la description et l'analyse des situations de travail vécues s'effectuent au moyen du langage. C'est aussi le moyen par lequel il rédige son dossier. Du point de vue du conseiller, le langage constitue le moyen par lequel il va guider le candidat dans l'analyse des situations de travail qu'il évoque, expliquer les attentes de la démarche, du dossier, et engager le candidat dans la préparation à la situation d'évaluation avec le jury.

Prendre pour objet d'analyse le développement d'un discours d'expérience sur le travail en situation d'accompagnement VAE suppose de s'intéresser notamment à la fonction du dialogue dans l'activité à partir de la manière dont le candidat et le conseiller l'instrumentent dans la situation d'entretien (Rabardel, 1999). Notre hypothèse pose que la source du développement de la pensée et du discours sur le travail pour le candidat réside dans le caractère polyfonctionnel du dialogue en situation d'accompagnement VAE (Kostulski, 2012). On étudie donc le rôle du dialogue dans les processus de transformation de la pensée et du discours sur le travail. L'objectif de la recherche visait à caractériser d'une part les modalités dialogales par lesquelles le discours et la pensée du candidat sur son travail se développent dans les interactions d'accompagnement ; d'autre part, de repérer des traces de ces dynamiques de développement dans les discours d'expérience produits par le candidat sur son travail. 
La recherche s'est centrée sur une analyse des dialogues qui prennent place dans les interactions entre conseillers et candidats au cours des entretiens d'accompagnement. A partir de cet axe de travail, nous avons cherché à comprendre en quoi l'activité dialogale dans les interactions d'accompagnement peut être instrumentée comme une forme de médiation et d'étayage aux dynamiques développementales de la pensée du candidat sur son expérience de travail. Les modalités par lesquelles le discours et la pensée du candidat s'élaborent ont été caractérisées à partir des mouvements dialogiques (voix mises en scène), relationnels (places endossées), contextuels (reconfigurations du cadrage) et interprétatifs (transformations des figures d'action) (Rémery, 2015). Ces différents mouvements ont permis de repérer des dynamiques de développement des discours d'expérience produits par le candidat au travers du dialogue. L'articulation de ces différents mouvements dans les analyses effectuées constitue une tentative théorico-méthodologique d'appréhender l'activité qui préside au développement d'un discours d'expérience du candidat sur son travail dans sa dimension processuelle et dynamique. Notre démarche d'analyse des données explore à la fois la dimension référentielle, discursive et interactionnelle des discours pour s'intéresser non seulement aux contenus et aux formes des discours d'expérience mais aussi aux voies de leur développement dans les interactions dialogales avec le conseiller. Cette perspective, centrée sur les dimensions interactionnelles, introduit des outils qui sont de nature à nous permettre de comprendre comment, dans la séquentialité des échanges entre le conseiller et le candidat, les énoncés d'expérience du candidat se transforment et rendent compte d'un possible développement professionnel.

\section{Repérage des mouvements dialogiques, relationnels, contextuels et interprétatifs à partir d'un extrait d'entretien d'accompagnement en VAE}

Trois conseillères ont été suivies au cours des entretiens qu'elles ont menés avec plusieurs candidats. Au total vingt-sept entretiens d'accompagnement individuel ont été enregistrés à partir desquels un travail de retranscription a été effectué. Nous proposons ici un extrait sélectionné pour le caractère significatif des mouvements dialogiques, relationnels, contextuels et interprétatifs repérables dans la séquentialité des échanges. Cet extrait a été délimité en fonction de l'ouverture et de la clôture d'une séquence thématique explorant une situation de travail vécue par le candidat. L'extrait proposé ci-dessous met en scène un binôme candidat/conseillère. Le candidat, Doris, est éducateur dans un club de prévention. L'action éducative des clubs de prévention vise à faciliter l'insertion sociale des jeunes par leur libre adhésion en collaboration avec les services sociaux, les établissements socio-éducatifs, culturels et sportifs. Les éducateurs en prévention se consacrent à un travail de rue en privilégiant les lieux où circulent les jeunes (bas d'immeubles, salles de jeux, cafés, alentours des établissements scolaires, stades et autres lieux publics). Doris ne possède pas de diplôme dans le travail socio-éducatif et souhaite obtenir le diplôme d'état d'éducateur spécialisé sur la base de cette expérience. La conseillère, Florence, est assistante sociale de formation. Elle est aujourd'hui responsable du dispositif VAE et accompagne aussi des candidats dans leur démarche de VAE. Dans l'extrait proposé, le candidat et sa conseillère discutent d'une situation de travail qui fera l'objet d'une présentation dans le dossier en relation à la fonction 1 du référentiel «Etablissement d'une relation, diagnostic éducatif ». Pour cette fonction, le candidat doit notamment faire la preuve de la façon dont il s'approprie les informations concernant la personne prise en charge, établit une relation éducative avec elle et élabore un diagnostic éducatif qui préfigure le projet qu'il va mettre en place. Dans l'extrait, le candidat présente pour la première fois une situation de travail au cours de laquelle il est entré en relation avec un groupe de jeunes.

\subsection{L'explicitation d'une situation de travail singulière}

Le début de l'extrait marque l'ouverture par la conseillère d'une séquence thématique sur l'exploration d'une situation de travail vécue par le candidat.

1. Acc: Bon... Alors donc on va commencer à parler peut-être de votre travail, hein? (hum) Donc si on retient l'idée - en tout cas pour l'instant, on verra ce qu'on fait après de cette idée - qu'il y aurait quatre situations se référant chacune à une fonction du référentiel, est-ce que vous avez par exemple par rapport à la fonction 1 une idée de ce dont vous pourriez parler?

Au plan référentiel, la conseillère propose au candidat en (1) d'investir deux espaces discursifs, celui du travail socio-éducatif (alors on va commencer à parler peut-être de votre travail ?) et celui de l'écriture du dossier (est-ce que vous avez par exemple par rapport à la fonction 1 une idée de ce dont vous pourriez parler ?). Cette proposition rend manifeste la façon dont elle endosse sa place d'accompagnatrice dans l'entretien. L'orientation proposée par la conseillère met ainsi le candidat en situation de penser l'articulation entre les fonctions du référentiel qui structure le dossier, et les situations de travail vécues (quatre situations se référant chacune à une fonction du référentiel). En effet, les situations de travail analysées par le candidat dans 
son dossier doivent être variées et de nature à démontrer les compétences attendues pour chacune des fonctions du référentiel du diplôme.

Le candidat entreprend en (2) la présentation d'une situation de travail dont il envisage de parler dans son dossier. II s'engage dans cette séquence ouverte par la conseillère sur l'exploration d'une situation de travail.

2. Cand: Dans la fonction 1, ce dont je pourrais parler justement, c'est comment j'suis rentré... Ba moi, mon arrivée dans ce club de prévention en fait. On m'a expliqué en fait que sur le quartier où j'allais travailler, y'avait justement un groupe de jeunes qui foutait un peu... qui foutait vraiment la zizanie et qui se faisait vraiment entendre jusqu'aux réunions des élus. Donc voilà, moi ça m'a plu. Justement parce que c'était une tranche d'âge avec laquelle j'aime bien travailler, c'est-à-dire les 14-17. Donc c'est pour ça que je voulais en parler dans la fonction 1. $C^{\prime}$ est comment j'ai fait pour rentrer dans ce groupe-là. Donc c'est ça.

C'est d'abord la voix du candidat de l'écriture du dossier VAE qui s'exprime (dans la fonction 1, ce dont je pourrais parler justement). II parle alors d'une place de candidat. Le candidat investit à ce titre l'espace de l'écriture du dossier sur un mode hypothétique (emploi du conditionnel : je pourrais). L'investissement de l'espace de la production du dossier VAE conduit le candidat à se déplacer progressivement vers l'espace du travail socio-éducatif. L'agir-référent se transforme de l'écriture du dossier au travail socio-éducatif avec la mise en scène de la voix de l'éducateur (mon arrivée dans le club de prévention... on m'a expliqué en fait... où j'allais travailler...moi ça m'a plu...). Son énoncé prend la forme d'un récit d'activité au passé dont les caractéristiques rendent compte de la figure de l'action-événement passé. On trouve aussi dans cet énoncé des traces d'un déplacement vers un niveau de description de l'activité de travail plus commentatif (c'est une tranche d'âge avec laquelle j'aime bien travailler). Ce déplacement permet au candidat d'apporter des arguments au choix de cette situation pour son dossier (donc c'est pour ça que je voulais en parler) et donc de basculer à nouveau dans l'espace de la production du dossier. Finalement, à l'intérieur d'un même tour de parole, le candidat peut parler de différentes places, comme nous pouvons l'observer ici, d'une place de candidat et d'une place d'éducateur. Ces changements de places peuvent s'observer à partir d'une analyse des mouvements dialogiques (voix mises en scène) qui rendent compte également de mouvements au niveau contextuel (changement de cadres). Ces mouvements contextuels marquent une transformation de la nature de l'engagement du candidat dans l'activité, soit le candidat est tourné vers la description d'une situation de travail, soit il est tourné vers la construction du dossier.

Le récit d'activité du candidat se poursuit à partir de plusieurs relances faites par la conseillère comme on peut le remarquer dans la suite de l'échange.

3. Acc: Alors comment vous avez fait? Racontez-moi un peu

4. Cand: Ba en fait, à mon arrivée en fait, mon collègue partait. C'est avec lui que je suis rentré dans le quartier.

5. Acc : Il partait, c'est-à-dire vous arriviez vous pour le remplacer?

6. Cand: Voilà

7. Acc : D'accord

8. Cand: Donc je suis rentré avec lui en faisant un tour du quartier. J'avais commencé à faire le tour du quartier tôt le matin. J'avais demandé. Moi, je préférais que ce soit le matin, justement du fait qu'on avait déjà parlé de ce groupe-là. Donc moi, déjà dans ma tête, c'était ma priorité. C'était la priorité. Ils m'avaient dit en fait « de par votre expérience, nous on a un groupe qui nous pose problème dans ce quartier ». Donc moi après, quand j'ai vu mon collègue, je lui ai demandé si ça ne le dérangeait pas de venir faire le tour du quartier tôt le matin. C'est comme ça que j'ai commencé et justement c'est là que j'ai commencé à les voir, dès le premier jour.

9. Acc: Alors ? Votre première réaction, c'était quoi quand vous avez vu ces jeunes, ce quartier?

10. Cand: Ma première réaction c'était que même si ... Dans ma tête, j'avais un pressentiment.

11. Acc: Ah bon? (rires)

Les relances de la conseillère en (3) (Alors comment vous avez fait ? Racontez-moi un peu), en (5) (Il partait, c'est-à-dire vous arriviez vous pour le remplacer ?) et en (9) (Alors ? Votre première réaction, c'était quoi quand vous avez vu ces jeunes, ce quartier?) permettent de maintenir l'orientation du candidat dans l'espace du travail socio-éducatif et de le convoquer à sa place d'éducateur. Elles orientent le candidat vers la construction d'un discours d'expérience produit à partir du « je » éducateur, et basé sur l'évocation d'une situation de travail singulière (comment vous avez fait ?... vous arriviez pour le remplacer ?... votre première réaction, c'était quoi...). Le candidat évoque le départ prochain du collègue qu'il va remplacer et avec lequel il se trouve engagé dans la situation évoquée. Son récit d'activité est construit à travers un discours dont l'énonciateur est fortement impliqué (je suis rentré... j'avais commencé... j'avais demandé... je préférais... quand j'ai vu mon collègue, je lui ai demandé... c'est comme ça que j'ai commencé... c'est là que j'ai commencé à les voir...). C'est à la fois ses actions mais aussi ses pensées qui sont mises en scène en (8) (je préférais... moi dans ma tête, c'était ma priorité) ou en (10) (dans ma tête j'avais 
un pressentiment). La présence de marqueurs temporels (à mon arrivée ; après) et le recours aux formes verbales du passé (passé composé, imparfait) caractérisent la figure de l'action-événement passé.

Dans la suite du récit, le candidat continue d'investir l'espace du travail socio-éducatif à travers un tour de parole relativement développé.

12. Cand: J'avais un pressentiment parce que je me suis dit que je vais faire le pari justement que d'y aller à cette heure-ci, parce que je sais pas... J'avais le pressentiment que j'allais les trouver à ce moment-là. Je sais pas si c'est un pur hasard ou une coïncidence mais poum, ce matin-là, je les ai vus. Et moi, je l'avais pas dit à mon collègue en fait. Ça veut dire que quand on a fait le tour du quartier, on les a vus. Avec mon collègue, on est allés les voir. Lui, il les connaissait un peu mais pas trop. Donc on a commencé à discuter avec eux. Donc il m'a présenté. Donc après, la discussion s'est faite comme ça, polie. Ils me posaient des questions. "T'habites où ? Ça fait combien de temps que t'es là ? Tu remplaces qui ? ». Donc le fait que j'ai dit que je remplaçais Alexandre, mon collègue qui va partir, ils m'ont dit... Enfin ils ont posé déjà la question à mon collègue parce qu'ils ne le voyaient pas souvent. "Depuis combien de temps t'es là et pourquoi tu pars? ». II leur a expliqué justement qu'il avait trouvé un poste " qui ne se refuse pas ". II leur a fait (rires). Donc après, ils en sont venus à moi. Ils m'ont posé des questions. Où je travaillais avant? J'habite où ? Donc j'ai commencé à parler avec eux. Au fil de la discussion - moi je connaissais un peu le quartier - et à ce moment-là, y'a quelqu'un qui passe. C'est quelqu'un que je connaissais mais qui travaillait à côté. Donc cette personne-là, elle s'est arrêtée. Elle m'a dit bonjour à l'écart. On a parlé cinq minutes. "Qu'est-ce que tu fais là ? » etc. et les petits m'entendaient. "Qu'est-ce que tu fais là ? Ba je travaille ici, c'est mon collègue ». Et y'avait un des jeunes qui connaissait cette personne-là. La personne en question me dit «attention à ces jeunes-là. Ils sont dangereux, ils font beaucoup de conneries, ils ont des problèmes avec la justice ». Alors j'ai dit " oui je sais t'inquiète pas. On est là pour ça ». Alors après je suis retourné avec les jeunes et ils m'ont dit «tu le connais lui ? Oui depuis l'enfance ». Donc direct, ils m'ont dit « toi tu dois connaître du monde? ". J'ai dit «non » mais après je leur explique que le quartier, c'est une plaque tournante. Moi j'suis de la banlieue, j'suis d'à côté donc je risque de connaître du monde. C'est de là, après la discussion, elle est partie. Ils m'ont posé des questions et il était 8 h30 alors je leur ai demandé s'ils n'allaient pas à l'école. Ils m'ont dit... C'était le mois d'avril, juste avant les vacances. J'ai dit "vous n'allez pas à l'école à cette heure-ci? Vous êtes matinal, 8h30, vous faites quoi ? ". Alors y'en a un qui m'a dit qui commençait à 10h, l'autre à 8 h30. J'ai dit « 8 h30 tu vas être en retard! ". II avait 15 ans alors je me suis dit qu'il devait être au collège en face. Il m'a dit qu'en ce moment il n'avait pas d'école. "Je me suis fait virer ». Après je leur ai demandé ce qu'ils allaient faire. En plus de ça, ils avaient fumé. Ils me posent la question si moi je fume. Je leur ai dit " c'est pas bon pour la santé ». Et je lui dis " et toi tu fumes depuis combien de temps? ". Sachant que le jeune en question il avait 16 ans. "Je sais pas » il me dit. "Qu'est-ce que ça t'apporte de fumer? Moi personnellement fumer, ça apporte rien, juste que des problèmes. Justement vu ton âge ». Et donc pour pas rentrer en conflit, moi je lui dis que " je suis pas là pour te faire la morale, c'est juste histoire de... moi je te connais pas assez, c'est juste que quand je vois des jeunes comme ça, moi ça me fait chier. Tu pourrais faire du sport, tu pourrais faire autre chose. Là tu fumes et tu sais même pas pourquoi. T'as l'air d'être un grand garçon, moi j'suis pas là pour te faire la morale, j'suis éducateur, on sera peut-être amené à faire des choses ensemble. Y'a pas de problème, si tu veux qu'on en discute, moi, y'a pas de problème ». Donc après on part. Je leur dis «à plus tard. Je vais faire un tour du quartier. Si vous voulez, venez avec moi comme ça vous me présentez le quartier ». Donc subitement y'en a un qui m'a dit " je rentre chez moi et je crois que je vais aller à l'école ». Je lui ai dit que c'était bien d'aller à l'école, c'est comme la santé. On se dit salut et il me dit qu'il espère qu'on fera des choses ensemble. Il en restait trois des jeunes. Ils sont restés avec nous. On a commencé à discuter, à parler. Je leur ai expliqué ce que je faisais avant, où je travaillais. Après ça s'est fait comme ça. Après ils sont tous rentrés chez eux (hum hum). On les a raccompagnés plus ou moins chez eux. Après j'étais avec mon collègue. On a commencé à discuter et je lui ai posé des questions sur ces jeunes-là. Après ça s'est fait comme ça.

Les pensées de l'éducateur agissant dans la situation de travail sont explicitées ( $\mathrm{J}$ 'avais un pressentiment parce que je me suis dit que je vais faire le pari justement que d'y aller à cette heure-ci, parce que je sais pas... J'avais le pressentiment que j'allais les trouver à ce moment-là) mettant en évidence un positionnement professionnel basé sur un registre intuitif. Le candidat décrit finement son activité mais aussi celle du collègue qui l'accompagne (on a fait le tour du quartier, on les a vus. Avec mon collègue, on est allés les voir. Lui, il les connaissait un peu mais pas trop. Donc on a commencé à discuter avec eux. Donc il m'a présenté). La conversation entre les éducateurs, le groupe de jeunes et un passant est mise en scène de façon alternée, entre récit (figure de l'action-événement passé) et discours rapporté. Les énonciateurs paraissent alors « incarnés » par le candidat à travers les différentes voix mises en scène. A travers son discours, le candidat donne à voir la construction qu'il opère pour 
la conseillère d'une certaine image de son rôle d'éducateur qui contribue à donner l'impression d'une figure de professionnel héros et sauveur (La personne en question me dit « attention à ces jeunes-là. Ils sont dangereux, ils font beaucoup de conneries, ils ont des problèmes avec la justice ». Alors j'ai dit « oui je sais t'inquiète pas. On est là pour ça »). Son discours permet aussi à la conseillère d'identifier les intentions de l'éducateur vis-à-vis des jeunes (Et donc pour pas rentrer en conflit) mais aussi le type d'informations récoltées sur la situation des jeunes à partir desquelles un diagnostic éducatif pourra être posé.

\subsection{La simulation de l'activité du jury}

Jusqu'à présent, nous avons identifié que la conseillère parlait plutôt d'une place d'accompagnatrice, cherchant à accompagner le candidat dans l'explicitation de la situation de travail. En (13) la conseillère transforme son engagement dans l'activité en prenant une place d'évaluatrice caractéristique de la posture du jury et en convoquant corrélativement le candidat à une place d'évalué.

13. Acc: Alors après ? Ça c'est la première étape que vous racontez là. On s'apprivoise. Après on sent qu'il y a quelque chose qui se construit. Comment on en arrive - parce que dans l'échange tel que vous le racontez - c'est plutôt vous faites connaissance mais vous n'êtes pas vraiment encore en position de l'éducateur qui propose un accompagnement pour des questions spécifiques. On n'en est pas encore là. Alors comment vous allez faire pour passer de cette première phase... ou plutôt comment vous avez fait pour passer de cette prise de contact, essayer qu'ils aient confiance en vous, à la phase suivante disons.

Ce basculement à une place de jury rend compte d'une transformation du cadrage de l'activité. Du point de vue de la conseillère, la situation semble prendre une autre signification que précédemment. Bien que le jury ne soit pas thématisé explicitement dans le discours, la conseillère s'engage ici dans une évaluation du travail de l'éducateur telle qu'elle pourrait être amenée à le faire en jury avec un autre candidat, en convoquant sa propre expérience d'évaluatrice et en mettant en scène la voix du jury (Ça c'est la première étape que vous racontez là. On s'apprivoise. Après on sent qu'il y a quelque chose qui se construit. Comment on en arrive - parce que dans l'échange tel que vous le racontez - c'est plutôt vous faites connaissance mais vous n'êtes pas vraiment encore en position de l'éducateur qui propose un accompagnement pour des questions spécifiques. On n'en est pas encore là). A cet égard, elle propose au candidat de se déplacer symboliquement et d'investir un nouvel espace d'activité, celui du jury, où la situation de travail racontée par le candidat va faire l'objet d'une évaluation. Cette évaluation permet de faire apparaître aux yeux du candidat deux étapes de son travail éducatif : une phase d' " apprivoisement » et une phase $d^{\prime}$ " accompagnement éducatif » à proprement parler. Elle met aussi en jeu une catégorisation identitaire qui force le candidat à se positionner (vous n'êtes pas vraiment encore en position de l'éducateur qui propose un accompagnement pour des questions spécifiques). L'énoncé de la conseillère se clôture par une question qui oblige le candidat à reconsidérer son travail socio-éducatif au prisme de ce découpage en deux phases (Alors comment vous allez faire pour passer de cette première phase... ou plutôt comment vous avez fait pour passer de cette prise de contact, essayer qu'ils aient confiance en vous, à la phase suivante disons).

Mis en situation d'évaluation fictive, le candidat reprend alors son récit en tentant d'apporter des éléments de justification à son action éducative.

14. Cand: Après en fait, comment... Après en fait, ce qui les a attirés et qui fait que j'ai pu construire quelque chose avec eux, c'est que en fait, on a la même passion avec eux. C'est le foot (hum hum). Un jour, je crois que c'était un samedi, je suis venu sur le quartier. Quand je suis arrivé, ils étaient mélangés. Les 18 étaient mélangés avec les 15-16. Donc ça faisait un groupe. Nous, notre objectif c'était de casser ce groupe (hum hum). Un jour, j'suis arrivé dans le square. La veille, j'avais parlé aux plus grands les 16-17 ans. Je leur avais parlé de foot. Donc le lendemain, quand je suis arrivé, on a discuté du match qui était passé à la télé (hum hum). Quand j'ai parlé de ça avec eux, déjà les petits ont écouté (hum hum). J'ai parlé du PSG (hum hum). En fait, quand j'ai parlé du PSG, j'ai dit « bon la semaine prochaine y'a un match au parc des princes, au pire des cas on va y aller. Je vais essayer de prendre des places et on va y aller ». Eux, ils ont entendu, mais ils ont rien dit (hum hum). Donc après ils ont attendu que je parte (hum hum) je les ai recroisés dix quinze minutes après (hum hum) et on a commencé à parler du parc. Donc c'est là que c'est parti.

15. Acc : D'accord.

16. Cand: Eux en fait, on va dire ce sont des fanatiques. A chaque match, au parc des princes, ils y vont (hum hum). Mais ils fraudent (hum hum). C'est comme ça, je le sais. Quand j'ai dit " moi la semaine prochaine, on va aller voir le match », ils m'ont dit "nous on y va ». "Quand vous y allez, vous payez vos places, c'est avec qui ? ". "On fraude. On y va, on fraude ». "Vous ne vous faites jamais attraper?". "Ba non, on connaît bien, le parc, c'est chez nous » (hum hum). Moi j'ai dit "c'est pas mieux si au lieu d'aller prendre des risques, on achète 
des places comme tout le monde pour regarder le match ». Ils m'ont dit " oui » mais ils m'ont dit qu'avant personne ne leur avait proposé (hum hum). Donc en fait c'est parti de là. Donc j'ai dit « ok je vais prendre les places"

Le candidat évoque une nouvelle situation avec les jeunes au cours de laquelle il leur a proposé d'aller à un match de foot. L'espace du travail socio-éducatif est investi, comme précédemment, au plan textuel et discursif par la production d'un énoncé caractéristique de la figure d'action-événement passé (récit interactif; implication agentive avec « je »; un ancrage temporel borné avec la présence de marqueurs « un jour », "c'était un samedi », « un jour », « la veille », « le lendemain », « la semaine prochaine »; des formes verbales au passé composé et à l'imparfait). La voix de l'éducateur est exprimée à certains moments restituant la conversation qu'il a eue avec les jeunes comme en (14) (j'ai dit « bon la semaine prochaine y'a un match au parc des princes, au pire des cas on va y aller. Je vais essayer de prendre des places et on va y aller ») et en (16) (Quand j'ai dit « moi la semaine prochaine, on va aller voir le match »...ils m'ont dit « nous on y va...). La reconstitution de ce moment de conversation avec les jeunes permet au candidat de dégager progressivement un moment important de la relation construite avec eux (Donc c'est là que c'est parti... Donc en fait c'est parti de là). Le récit fait aussi apparaître une nouvelle fois des prises d'informations au cours de la situation de travail (Quand je suis arrivé, ils étaient mélangés. Les 18 étaient mélangés avec les 15-16. Donc ça faisait un groupe) relatifs à des objectifs fixés préalablement avec l'équipe éducative (Nous, notre objectif c'était de casser ce groupe).

\subsection{La structuration de l'écrit VAE}

En (17), la conseillère transforme une nouvelle fois son engagement dans l'activité. Elle quitte sa place d'évaluatrice endossée précédemment pour une place d'accompagnatrice en se centrant sur la construction du dossier.

17. Acc : D'accord. Ok donc là, cette fonction 1, elle part chronologiquement de l'établissement de la relation. C'est-à-dire du moment où vous rencontrez les jeunes jusqu'au moment où vous construisez avec eux un pré-projet ou un projet, pour répondre à des besoins, des demandes qu'ils ont. Donc ça s'arrêterait à quel moment? Donc là, vous commencez l'histoire parc des princes etc., et après, ça va jusqu'où ? Vous pensiez vous arrêter à quel moment?

18. Cand: Avec eux ?

19. Acc: Non dans l'écrit que vous allez faire.

20. Cand: L'écrit?

21. Acc : L'écrit va jusqu'à quelle étape du travail que vous avez fait avec eux?

22. Cand: Je dirais que l'écrit porterait sur le moment où je les ai rencontrés jusqu'au moment où j'suis allé avec eux au match (hum hum).

La définition que projette la conseillère de la situation évolue vers l'investissement de l'espace du dossier. La conseillère centre son propos sur la structuration du récit en questionnant le candidat sur la délimitation chronologique de la situation de travail telle que le candidat envisage de la raconter dans son dossier (17) (Ok donc là cette fonction 1, elle part chronologiquement de l'établissement de la relation. C'est-à-dire du moment où vous rencontrez les jeunes jusqu'au moment où vous construisez avec eux un pré-projet ou un projet pour répondre à des besoins, des demandes qu'ils ont. Donc ça s'arrêterait à quel moment? Donc là, vous commencez l'histoire parc des princes etc., et après, ça va jusqu'où ? Vous pensiez vous arrêter à quel moment ?). Cette activité de structuration s'appuie sur le référentiel à partir duquel des termes professionnels sont repris mot pour mot tels que «l'établissement de la relation », «la construction d'un pré-projet », "répondre à des besoins ». A ce titre, on pourrait dire que la conseillère parle depuis la voix des référentiels. Le récit par le candidat de sa situation de travail est " découpé » selon les différentes étapes proposées dans le référentiel. La question de la conseillère invite le candidat à investir l'espace de la production du dossier en l'amenant à anticiper la question de la clôture du récit de cette situation de travail qu'il va écrire. L'étonnement manifesté en (18), rend visible que le candidat n'a pas compris le changement de cadre opéré par la conseillère (avec eux ?) et se situe dans l'espace d'activité investi précédemment, celui du travail socio-éducatif, ce qui oblige la conseillère à un recadrage de la situation en (19) (Non dans l'écrit que vous allez faire) et en (20) (L'écrit va jusqu'à quelle étape du travail que vous avez fait avec eux ?). Le candidat s'ajuste alors à la définition de la situation et s'engage dans l'espace de la production du dossier en (21) (Je dirais que l'écrit porterait sur le moment où je les ai rencontrés jusqu'au moment où j'suis allé avec eux au match). Cet ajustement le conduit à parler depuis la place du candidat engagé dans l'espace de l'écriture du dossier. La voix de l'éducateur cède la place à celle du candidat de l'écriture du dossier. 


\subsection{Alternance entre simulation de l'activité du jury et production de l'écrit}

A partir de (23), l'activité se reconfigure autour de la situation de travail du candidat. La conseillère transforme à nouveau le cadre de la situation d'accompagnement et engage le candidat dans la construction d'une argumentation de son positionnement éducatif

23. Acc: Mais ces jeunes, vous les aviez identifiés? C'était quoi la problématique de ce groupe ? C'était quoi votre champ où vous, vous pouviez envisager de les aider en tant qu'éducateur?

24. Cand: Mon champ, c'était de les occuper (hum hum) parce qu'en fait, c'était ça leur problème comme ils ne faisaient rien.

25. Acc : Alors pourquoi les occuper? Parce qu'a priori, c'est pas du travail d'éducateur occuper les jeunes?

26. Cand: Quand je dis les occuper, c'est en fait qu'ils ne fassent pas de conneries dans la rue (hum hum). C'est s'intéresser à eux (hum hum).

27. Acc: Alors pourquoi? Dans quel objectif? Vous avez une idée derrière la tête quand vous faites ça?

28. Cand: Oui mon idée, c'est qu'au final, ils retrouvent tous une structure, c'est-à-dire aller à l'école ou trouver une formation. C'est aussi qu'ils arrêtent leurs conneries

Par la question formulée en (23) (Mais ces jeunes, vous les aviez identifiés? C'était quoi la problématique de ce groupe? C'était quoi votre champ où vous vous pouviez envisager de les aider en tant qu'éducateur ?), la conseillère encourage le candidat à expliciter le diagnostic éducatif qu'il a posé sur le comportement des jeunes. Le recours à l'expression « en tant qu'éducateur » marque son intention d'insister sur le positionnement professionnel du candidat face à ces jeunes. II rend compte aussi d'une évaluation implicite qui supposerait que ce qu'a dit le candidat jusqu'à présent ne met pas en évidence, du moins de façon claire, les compétences d'un éducateur. A ce titre, on peut penser que par cette question, la conseillère parle depuis une place d'évaluatrice en engageant le candidat dans l'espace du jury. Elle convoque ainsi le candidat à une place corrélative d'évalué en l'amenant à produire une réponse qui pourrait être celle qu'il produirait face au jury. C'est ainsi la voix de l'éducateur qu'elle convoque dans le discours du candidat ou, plus précisément, celle qu'il tiendrait en tant qu'éducateur face au jury. En (24), le candidat se prête au jeu de l'évaluation et propose ainsi d'expliciter les besoins qu'il a identifiés justifiant son action éducative (Mon champ, c'était de les occuper parce qu'en fait, c'était ça leur problème comme ils ne faisaient rien). Parce que la réponse produite par le candidat succède à une évaluation et une demande d'argumenter de la part de la conseillère, on peut penser qu'elle constitue une forme de simulation de la discussion avec le jury. Cette simulation se poursuit jusqu'en (28). Notamment, en (25) et (27), la conseillère maintient son engagement dans l'espace du jury au sens où elle poursuit sur un questionnement qui sous-tend une posture évaluative de la pratique éducative du candidat (Alors pourquoi les occuper ? Parce qu'a priori, c'est pas du travail d'éducateur occuper les jeunes ?... Alors pourquoi ? Dans quel objectif ? Vous avez une idée derrière la tête quand vous faites ça ?). Quant au candidat, il précise en (26) et (28) la justification de son action éducative (Quand je dis les occuper, c'est en fait qu'ils ne fassent pas de conneries dans la rue. C'est s'intéresser à eux ... Oui mon idée, c'est qu'au final, ils retrouvent tous une structure, c'est-à-dire aller à l'école ou trouver une formation. C'est aussi qu'ils arrêtent leurs conneries). Les caractéristiques linguistiques des réponses produites par le candidat en (24), (26) et (28) rendent compte de la construction d'une figure de l'action définition dont l'agir-référent est l'action éducative (présence d'un discours théorique ; ancrage temporel non borné ; forme verbale à valeur générique) (Mon champ, c'était de les occuper... c'est en fait qu'ils ne fassent pas de conneries... C'est s'intéresser à eux... c'est qu'au final, ils retrouvent tous une structure... C'est aussi qu'ils arrêtent leurs conneries). Ces caractéristiques marquent l'investissement par le candidat d'une position plus argumentative sur son travail éducatif et contrastent avec les récits descriptifs produits précédemment qu'on avait repérés à partir des figures d'action-événement passé. Ces transformations au plan textuel et discursif de la façon de thématiser le travail socio-éducatif permettent de retracer le mouvement interprétatif engagé par le candidat sur son travail.

La réaction de la conseillère en (29) marque un décrochement par rapport à l'activité dans laquelle elle s'est engagée précédemment. Si elle maintient son orientation dans une position d'évaluation, elle s'engage aussi dans une explicitation des raisons de son évaluation et des conséquences au plan de l'écriture du dossier pour le candidat.

29. Acc: Vous voyez ça, il va falloir l'expliquer. Le fait d'être avec des jeunes - je caricature exprès ce que vous venez de dire - de leur proposer d'aller avec eux au Parc des Princes ou je ne sais pas quoi, on n'est pas forcément spécifiquement dans un travail d'éducateur si on le raconte comme ça (hum hum). On peut être dans un travail d'animateur de quartier. Où on est dans un travail d'éducateur, c'est à partir du moment où on identifie des problématiques qui sont spécifiques à ce groupe de jeunes et on commence à imaginer avec eux une manière de prendre en charge ces difficultés. Là, on est dans le travail éducatif. Mais ça suppose que ça, ça soit explicité dans votre écrit. Sinon, si vous n'expliquez pas que si vous allez avec eux au parc des princes, c'est parce que vous avez d'autres idées derrière la tête. C'est pour ça que c'est important de ne pas rester dans la description du faire, hein? Parce que ce n'est pas ce que vous faites (hum) qui va prouver à un jury que 
vous êtes éducateur. C'est pas parce qu'on va à un match avec des jeunes qu'on est éducateur. En revanche, si le fait d'aller à un match avec des jeunes, c'est une technique de travail pour construire de la relation, pour que les jeunes investissent des trucs qui leur font plaisir, pour progressivement être capable de construire un projet pour eux-mêmes. Ça c'est du travail éducatif. Ça suppose que tout ce travail invisible vous le mettiez en valeur dans le livret. C'est ça qui fait que c'est du travail d'éducateur. Donc y'a toujours cette idée qu'il va falloir que vous ayez en tête à chaque fois que vous écrirez une ligne dans ce livret, c'est qu'il faut bien expliciter vos intentions à vous quand vous faites quelque chose. Si vous les emmenez au parc des princes, c'est pas simplement pour le plaisir de les emmener au parc des princes, même si vous aimez le foot. C'est qu'il y a quand même un objectif éducatif qui est pour vous important. J'insiste là-dessus parce qu'on n'a pas forcément l'habitude d'écrire comme ça quand on écrit. Mais là, c'est ce qu'il va falloir faire. Ok?

Alors que le candidat était engagé au tour précédent dans la réponse qu'il pourrait produire au jury si la question de son champ d'intervention lui était posée par le jury, la conseillère amène à nouveau en (29) le candidat à se déplacer dans de l'écriture du dossier (vous voyez ça, il va falloir l'expliquer). Ce déplacement marque un changement de cadrage où est mise en scène la voix de l'accompagnatrice. L'énoncé porte les caractéristiques d'une modalisation déontique exprimée sur le registre de l'obligation (il va falloir) signalant au candidat la nécessité de donner à voir au jury, à travers l'écriture, certains aspects du travail. La réponse produite par le candidat est replacée dans l'espace de l'écriture du dossier comme ce qu'« il va falloir expliquer ». La suite du tour de parole présente une forte instabilité au plan dialogique, et corrélativement au plan contextuel, au sens où la conseillère transforme, d'un énoncé à l'autre, son engagement dans l'activité. On peut observer à cet égard un enchaînement alterné de voix mises en scène entre celle de l'évaluatrice-experte du champ éducatif et celle de l'accompagnatrice à l'écriture du dossier. Ces aller-et-retours entre la voix de l'évaluatrice-experte et celle de l'accompagnatrice rendent compte à la fois de mouvements continus au niveau des places énonciatives endossées et de changements de cadrages, alternant entre l'investissement de l'espace du jury et de celui de l'écriture du dossier. A travers la voix de l'évaluatrice-experte, la conseillère s'engage dans une évaluation du récit du candidat (on n'est pas forcément spécifiquement dans un travail d'éducateur si on le raconte comme ça... C'est pas parce qu'on va à un match avec des jeunes qu'on est éducateur). Elle définit (figure de l'action-définition) aussi ce qu'est le travail éducatif par opposition à un travail d'animation de quartier (Où on est dans un travail d'éducateur, c'est à partir du moment où on identifie des problématiques qui sont spécifiques à ce groupe de jeunes (...) Ça c'est du travail éducatif). A travers la voix de l'accompagnatrice, la conseillère formule des recommandations pour l'écrit du dossier VAE (ça suppose que ça, ça soit explicité dans votre écrit... C'est pour ça que c'est important (...) on n'a pas forcément l'habitude d'écrire comme ça quand on écrit. Mais là, c'est ce qu'il va falloir faire). Ces recommandations prennent un caractère d'obligation (ça suppose que... il va falloir... il faut bien expliciter... c'est ce qu'il va falloir faire). Chacune des places endossées par la conseillère convoque le candidat à une place corrélative, soit de candidat-évalué, soit de candidat engagé dans l'écriture du dossier.

Nous avions repéré précédemment en (24), (26) et (28) que le candidat commençait, à travers la voix de l'éducateur, à adopter un positionnement moins descriptif en s'engageant dans la justification de son action éducative. En (30), (32) et (34), ce positionnement se précise davantage et permet de suivre le mouvement interprétatif du candidat à propos du travail éducatif.

30. Cand: Oui c'est pour ça en fait, la sortie. C'était pour sortir du cadre, du quartier. Les langues ont pu se délier plus facilement. Du fait qu'on sorte du quartier, on a pu parler d'autre chose. Moi j'ai pu en apprendre un peu sur leur personnalité (hum hum). Le fait de les mettre hors du quartier, c'était aussi pour moi voir comment ils se comportaient hors du quartier.

31. Acc: Hum hum bien sûr.

32. Cand: C'était aussi pour ça. J'attendais aussi un retour d'eux justement par rapport à cette sortie-là. Après justement, j'ai pu les prendre en individuel, parce que quand je les voyais en collectif, c'était une discussion collective (hum hum). Je ne parlais pas à une seule personne, je parlais au groupe. Quand je leur parlais de certaines choses, ils me posaient la question comment je le savais. Alors je leur disais " attendez je suis éducateur quand même (hum hum). Tout se sait dans un quartier. Je n'ai pas besoin d'être à côté de vous pour savoir ce que vous avez fait. Vous savez, quand on fait quelque chose de grave, vous savez très bien que ça va se savoir ». Après, ça c'est fait comme ça. Eux ils savaient que je savais ce qu'ils faisaient (hum hum). Donc je travaillais en individuel quand je les croisais, mais souvent à certaines périodes de la journée, ils étaient ensemble donc je parlais justement. Là on parlait.

33. Acc: D'accord, donc il va falloir mettre en évidence les compétences du référentiel. Il va falloir montrer comment vous avez construit la relation. Qu'est-ce que vous avez observé chez ces jeunes? Ce que vous avez observé, comment vous l'avez analysé, qu'est-ce que vous en avez déduit, et à partir de là, quelles actions vous voulez mettre en place pour répondre aux problèmes que vous avez repérés. Le travail de VAE, c'est 
de déconstruire. Alors c'est vrai qu'à partir du moment où on le fait, on n'a pas forcément toutes les étapes, comment on voyait par exemple, on observe, on analyse tout ça. Tout ça, on ne l'a pas forcément, on ne se dit pas forcément premièrement j'observe, deuxièmement j'analyse. Tout ça, on le fait sans s'en rendre compte. Le travail VAE, c'est justement ce travail de déconstruction parce que c'est ça qui fait la compétence professionnelle finalement. C'est pas tant le résultat, parce que le résultat, si le résultat c'est aller au parc des princes c'est pas très compliqué à faire encore que moi je ne sais pas trop comment on prend les places et tout ça (rires) - je devrais pouvoir trouver si je m'en occupais - mais la difficulté c'est tout le reste.

34. Cand: Oui en fait on va dire aller voir le match avec eux, c'était en fait rentrer dans le groupe.

35. Acc: Voilà, ba c'est tout ça qu'il va falloir nous dire.

Le candidat apporte de nouvelles justifications à la sortie au parc des princes avec les jeunes en (30) (oui c'était pour ça en fait la sortie. C'était pour sortir du cadre, du quartier...C'était aussi pour moi voir comment ils se comportaient hors du quartier), en (32) (C'était aussi pour ça. J'attendais aussi un retour d'eux justement par rapport à cette sortie-là), en (34) (en fait on va dire aller voir le match avec eux, c'était en fait rentrer dans le groupe). Ces justifications prennent la forme d'une succession de figures d'action-définition qui soulignent les mouvements interprétatifs opérés par le candidat, à la fois au plan de son engagement dans un positionnement plus argumentatif sur le travail éducatif et au plan thématique dans la précision de ses intentions éducatives. En (33) et (35), la conseillère se déplace vers l'espace de l'écriture du dossier VAE en parlant à nouveau depuis une place d'accompagnatrice. Le registre déontique est privilégié (donc il va falloir mettre en évidence les compétences du référentiel. Il va falloir montrer ... c'est tout ça qu'il va falloir nous dire). Nous pouvons repérer ici la trace d'un travail de définition proposé au candidat pour l'accompagner dans la compréhension de la démarche d'écriture du dossier (Le travail de VAE, c'est de déconstruire. ... c'est justement ce travail de déconstruction parce que c'est ça qui fait la compétence professionnelle finalement. C'est pas tant le résultat...).

\section{Transformations d'engagement des acteurs et dynamiques développementales du discours d'expérience du candidat dans l'interaction}

\subsection{Complexité et hétérogénéité des interactions en situation d'accompagnement VAE}

L'analyse de l'extrait a permis de faire apparaître différents moments de l'entretien dans la séquentialité des échanges entre le candidat et la conseillère qui sous-tendent une transformation du cadre de l'activité (Goffman, 1991). Plusieurs cadrages ont été identifiés tels que : I'explicitation d'une situation de travail singulière, la simulation de l'activité du jury VAE, la structuration ou la production de l'écrit VAE. Ces transformations reconfigurent la nature de l'activité en cours et permettent de retracer la trajectoire d'engagement du candidat et de la conseillère. Ainsi le candidat est amené à s'engager successivement dans différentes activités telles que : la description d'une situation de travail singulière, l'argumentation de son positionnement éducatif et la structuration de son écrit. La conseillère s'engage elle aussi dans une succession d'activités telles que : I'accompagnement à la description d'une situation de travail par le candidat, l'évaluation du positionnement éducatif du candidat, l'accompagnement à la structuration de l'écrit du candidat et la formulation de conseils pour l'écrit VAE.

Chacune des activités engage le candidat ou la conseillère à faire parler différentes voix énonciatives (Ducrot, 1984; Vion, 1998). La voix de l'éducateur ou du candidat engagé dans l'écriture s'exprime ainsi alternativement à travers le discours du candidat. De même la voix de l'accompagnatrice ou du jury s'exprime à travers le discours de la conseillère. A partir des voix mises en scène dans les discours de la candidate et de la conseillère émergent des espaces d'activité tels que l'espace du travail socio-éducatif, l'espace de l'écriture du dossier ou l'espace du jury. L'entretien d'accompagnement se caractérise donc par des mouvements de nature dialogique qui correspondent aux voix énonciatives mises en scène par le conseiller et le candidat. Nous avons caractérisé ces voix à partir des expériences convoquées dans et par les discours du conseiller et du candidat et avons pu constater qu'à l'intérieur même de l'interaction constitutive de l'entretien d'accompagnement VAE se jouent des interactions simultanées mettant en jeu d'autres dialogues (Vion, 2008). L'entretien VAE apparaît ainsi comme un lieu de cristallisation de dialogues avec des personnes qui ne sont pas présentes mais auxquelles le candidat ou le conseiller peuvent se référer ou dont ils peuvent invoquer les discours plus ou moins explicitement à partir des voix mises en scène dans leurs discours. S'enchâssent alors sur le dialogue entre le conseiller et le candidat d'autres dialogues, à savoir des dialogues dans le dialogue in situ, qui résultent des espaces d'activité investis.

Aussi, si le conseiller apparaît comme un « destinataire second » (Astier, 2001 ; Clot, 2005) de l'échange concret et immédiat de la situation d'accompagnement, c'est à travers lui que se mettent en place des dialogues « à distance » avec d'autres destinataires, qui participent au développement du discours d'expérience du candidat. C'est dire que l'entretien d'accompagnement VAE peut, comme tout échange, être étudié non seulement d'un point de vue dialogal mais aussi dialogique (Grossen \& Salazar-Orvig, 2006). II existe ainsi dans les entretiens d'accompagnement VAE que nous avons observés une " pluralité de 
destinataires » (Astier, 2005) aux discours des candidats et du conseiller, qui fait de l'accompagnement un espace polyphonique de discussion sur le travail. C'est précisément le caractère polyphonique (Ducrot, 1984) de l'entretien qui nous semble à même de valider l'hypothèse de travail formulée en amont de nos analyses. En effet, le développement d'un discours d'expérience du candidat sur son travail trouve son moteur ou ses ressources dans la pluralité des voix à partir desquelles ce dernier peut parler du travail et la pluralité des destinataires auxquels il peut s'adresser à travers le conseiller. Comme nous avons pu l'illustrer, le candidat peut s'adresser à des interlocuteurs différents en fonction de la dynamique des places endossées, des expériences convoquées et des voix mises en scène par le conseiller dans l'échange (une expérience éducative, une expérience d'accompagnement VAE, une expérience de jury). Astier (2005) souligne que "l'activité du sujet ne s'épuise pas dans la prise en compte de l'interlocuteur mais se définit en fonction de tous ceux que le message vise en s'adressant à son partenaire de l'interlocution » (p. 7). II importe donc d'identifier cette " pluralité des destinataires », c'est-à-dire les différentes situations d'interaction qui se jouent au cours de l'entretien en VAE et qui permettent aux candidats de développer un discours d'expérience sur le travail dans une pluralité d'adresses.

Dans l'extrait analysé, différents rapports de places (Vion, 1992 ; Flahault, 1978) ont été identifiés selon les espaces d'activité investis. L'accompagnement en VAE est une interaction spécialisée dans laquelle le rapport de places se trouve d'emblée préétabli en termes de rôles sociaux institutionnels : le conseiller VAE intervient en tant qu'accompagnateur, c'est-à-dire de sa position professionnelle; le candidat se trouve, lui convoqué à une place corrélative d'accompagné. Ces rapports institutionnels contribuent à définir le cadre social de la rencontre et justifient une certaine typologie des interactions et des engagements réciproques qui y prennent place. Cette typologie fonctionne comme une sorte d'implicite, un cadre d'expérience primaire au sens de Goffman (1991), qui permet aux participants de s'y engager de façon adéquate. Tout en postulant la stabilité du cadre interactif tel que défini par Vion (1992), c'est-à-dire un rapport social et institutionnel dominant préétabli entre le conseiller et le candidat qui se maintient tout au long de l'interaction, il est possible de penser la complexité et l'hétérogénéité des interactions en situation d'accompagnement VAE à partir de l'investissement d'espaces d'activité (l'espace de l'écriture du dossier, l'espace du travail socio-éducatif, l'espace du jury) qui rendent compte d'une transformation dynamique des rapports de places énonciatives et des voix mises en scène. Le cadre interactif de l'entretien d'accompagnement VAE ne présuppose pas la mobilisation constante et univoque d'un seul type d'interaction, que l'on pourrait rapporter à un seul type de rapport de places, dans l'accomplissement même de l'activité. Comme l'a souligné Vion (2008), «il convient de pouvoir penser, dans le même mouvement, la permanence du cadre interactif (...) et la variabilité des types d'interactions localement mobilisés » (p. 33). Les analyses ont justement mis en évidence le caractère hétérogène et dynamique des types d'interactions qui se développent au cours d'un entretien d'accompagnement.

Différentes configurations prennent place au cours du dialogue entre le conseiller et le candidat - accompagnateur/ accompagné, évaluateur/évalué comme dans l'extrait étudié mais aussi éducateur/éducateur comme nous avons pu le montrer par ailleurs (Rémery, 2013) - mettant en évidence une trajectoire d'engagements pluriels des acteurs dans l'activité. Ces configurations apparaissent comme une source d'étayage au développement de la pensée et du discours du candidat sur son travail. Ces configurations se manifestent par des « voix » mises en scène qui convoquent des expériences passées, anticipées ou imaginées, associées à des espaces d'activité distincts de la situation d'accompagnement (le travail, l'écriture du dossier, le jury). Parce qu'elles permettent au candidat de s'engager dans des « dialogues fictifs » reconfigurant les places endossées par chacun, ces configurations permettent d'appréhender les mouvements interprétatifs du candidat et rendent possible une transformation du point de vue porté sur l'activité professionnelle. Un « jeu » s'installe alors entre conseiller et candidat dans "l'espace protégé » de l'entretien d'accompagnement au sein duquel s'organise et s'expérimente une variation des contextes d'énonciation de l'expérience. Ceux-ci favorisent le développement de différentes formes de discours d'expérience sur le travail qui sont susceptibles d'être réinvestis dans les activités cible auxquelles l'accompagnement prépare (l'écriture du dossier et la confrontation avec le jury). Les variations d'adresse fictive induisent des remaniements au niveau de l'activité du candidat. Celui-ci est alors conduit à regarder son activité de travail par les yeux d'un autre. Ce sont là autant d'occasions de configurer son activité de travail par le discours en privilégiant, à chaque fois, un certain point de vue. Si le développement d'un discours d'expérience sur le travail chez le candidat est amorcé par la pluralité des destinataires auxquels il s'adresse, alors il importe de construire des situations d'accompagnement qui favorisent cette variation des adresses. En tant que ressources de l'activité du conseiller et du candidat, ces configurations apparaissent donc comme un instrument dont I'appropriation par les uns et les autres se présente comme centrale en situation d'accompagnement VAE. A ce titre, il pourrait constituer un outil d'analyse de l'activité précieux à la formation des conseillers.

\subsection{Des genèses interprétatives de l'activité au développement professionnel ?}

Quels liens peut-on établir entre ces processus de transformation du discours d'expérience et des processus plus larges de développement professionnel ? En cherchant à comprendre comment le candidat rend compte de situations de travail vécues, 
nous avons porté notre attention sur les transformations de son discours d'expérience, c'est-à-dire sur la manière dont des interprétations de l'activité de travail du candidat sont produites, partagées et reconfigurées avec l'aide du conseiller. Les caractéristiques linguistiques des figures d'action mises en évidence par le groupe de recherche LAF (Bronckart \& Bulea, 2006 ; Bulea, 2007) ont constitué à ce titre un outil précieux d'identification du point de vue adopté par le candidat pour configurer des représentations de son activité de travail. Nous avons mis en évidence que l'activité de travail peut être appréhendée de différentes façons par le candidat dans son discours et que cette appréhension rend compte d'une activité interprétative qui engagent des représentations de l'activité de travail, des représentations de soi dans l'activité de travail, des représentations de la prescription, des représentations des usagers, des collègues, de la hiérarchie, de l'environnement de façon générale. Le repérage successif de figures d'actions variées permet de retracer a posteriori une genèse interprétative de l'activité de travail dans le discours du candidat. Cette activité interprétative relève à la fois d'une activité de pensée et d'une activité discursive. A ce titre, le discours d'expérience rend compte à la fois d'une pensée et d'un discours sur l'activité de travail, construite par et pour le sujet.

L'objectif des analyses visait moins à délimiter la conception que le candidat se fait de son métier que comprendre la manière dont le candidat réélabore, au fil des interactions avec le conseiller, des représentations du travail susceptibles de contribuer au développement d'un discours d'expérience. C'est donc la pensée au travail ou la pensée en mouvement dans les échanges que nous avons tenté de saisir. L'analyse des interactions dialogales joue un rôle capital dans la compréhension de ces phénomènes puisque c'est au fil d'échanges et de débats entre le candidat et le conseiller, qui les amènent à endosser successivement différentes places, à mettre en scène différentes voix, à convoquer différentes expériences, que progressivement évoluent la pensée et le discours du candidat sur son activité de travail. Nous avons de ce fait montré comment la dynamique interactionnelle de l'entretien, mettant en scène un conseiller et un candidat partageant l'exercice d'un même métier, permet de favoriser la mise en mots des éléments significatifs d'une expérience professionnelle singulière vécue par le candidat, mais également d'amorcer une argumentation du positionnement de l'acteur dans cette situation (ses intentions, ses motifs, ses objectifs, son diagnostic, etc.) ou une généralisation des enjeux de celle-ci. Nous pensons que ces mouvements interprétatifs, visant à articuler la description d'une expérience de travail dans sa dimension singulière, argumentative et généralisée, engagent le candidat dans des déplacements de pensée sur son travail repérés à travers son discours qui participent, de notre point de vue, d'un développement professionnel.

On peut toutefois apporter quelques limites à ces conclusions qui ouvrent des questions et des perspectives de recherches futures. Les genèses interprétatives ont été retracées à partir d'extraits sélectionnés relativement courts. C'est là probablement une limite du travail en ce que les déplacements repérés sont circonscrits sur une temporalité très réduite. En même temps, le type d'analyse déployée ne nous permettait pas de nous engager sur des analyses à l'échelle d'un entretien complet. Le repérage de déplacements de pensée au plan du discours et des interactions ne peut s'observer et s'argumenter sans une démarche d'analyse qui reste très proche du "tissu discursif », de ses «pulsations » et "fluctuations » au fil de l'interaction (Vion, 1998). La saisie de mouvements interprétatifs dans une perspective longitudinale, à l'échelle d'un entretien, voire d'un parcours d'accompagnement complet, reste une question méthodologique complexe à élucider pour la recherche, d'autant plus si l'on souhaite appréhender des processus de développement professionnel induits par le dispositif dans son ensemble.

\section{Références bibliographiques}

Astier, P. (2001). La communication de l'expérience. Éléments d'analyse de l'activité du sujet. (Thèse de doctorat inédite). Conservatoire National des Arts et Métiers de Paris.

Astier, P. (2005). Dire, faire et savoir. Remarques sur leurs relations à l'occasion des " discours d'expérience ». Gème Congrès Européen de Science des Systèmes, Brest, 19-22 septembre 2005.

Barbier, J.-M. (2006). Le modèle francophone de la VAE. Sciences Humaines, 175, 10-11.

Bronckart, J.-P. \& Bulea, E. (2006). La dynamique de l'agir dans la dynamique des discours. Dans J.-M. Barbier \& M. Durand (Dir.), Sujet, activité, environnement : approches transverses (p. 105-134). Paris : Presses Universitaires de France.

Bulea, E. (2007). Le rôle de l'activité langagière dans l'analyse des pratiques à visée formative. (Thèse de doctorat inédte). Université de Genève.

Clot, Y. (2005). L'auto-confrontation croisée en analyse du travail : I'apport de la théorie bakhtinienne du dialogue. In L. Filliettaz \& J.-P. Bronckart (Dir.), L'analyse des actions et des discours en situation de travail. Concepts, méthodes et applications (p. 37-55). Louvain-la-Neuve : Peeters.

Ducrot, O. (1984). Le dire et le dit. Paris : Éditions de Minuit.

Flahault, F. (1978). La parole intermédiaire. Paris : Le Seuil.

Goffman, E. (1991). Les cadres de l'expérience. Paris : Éditions de Minuit.

Grossen, M. \& Salazar-Orvig, A. (2006). L'entretien clinique. Analyse des interactions verbales d'un genre hétérogène. Paris: Belin. 
Kostulski, K. (2012). La diversité fonctionnelle du langage : usage et conflictualités dans l'activité. Dans Y. CLOT (dir.), Vygotski maintenant (p. 237-254). Paris : La Dispute.

Salazar-Orvig, A. \& Grossen, M. (2008). Le dialogisme dans I'entretien clinique. Langage et Société, 123, 37-52.

Rabardel, P. (1999). Le langage comme instrument? Éléments pour une théorie instrumentale étendue. Dans Y. CLOT (Dir.), Avec Vygotski (p. 241-264). Paris : La Dispute.

Rémery, V. (2015). Développer un discours d'expérience sur le travail. Contribution à une analyse des discours et des interactions en situation d'accompagnement à la Validation des Acquis de l'Expérience. (Thèse de doctorat inédite). Conservatoire National des Arts et Métiers de Paris.

Rémery, V. (2013). Approche interactionnelle des processus de développement professionnel en accompagnement à la Validation des Acquis de l'Expérience. Les Sciences de l'Education Pour l'Ere Nouvelle, 46(4), 47-68.

Vion, R. (1992). La communication verbale. Analyse des interactions. Paris : Hachette.

Vion, R. (1995). La gestion pluridimensionnelle du dialogue. Cahiers de linguistique française, 17, 179-203

Vion, R. (1998). De l'instabilité des positionnements énonciatifs dans le discours. Dans J. Verschueren (Dir.), Pragmatics in 1998 : Selected papers from the 6th International Conference (vol.2) (pp. 577-589). Antwerp : International Pragmatics Association.

Vion, R. (1999). Pour une approche relationnelle des interactions verbales et des discours. Langage et société, 87, 95-114.

Vion, R. (2008). De la complexité des positionnements dans I'interaction verbale. Dans R. Delamotte-Legrand, C. Hudelot \& A. Salazar Orvig (dir.), Dialogues, mouvements discursifs, significations (p. 31-48). Paris : Éditions Modulaires Européennes. 